\title{
Interleaved Core Assignment for Bidirectional Transmission in Multi-Core Fibers
}

\author{
Ye, Feihong; Morioka, Toshio
}

Published in:

39th European Conference and Exhibition on Optical Communication (ECOC 2013)

Publication date:

2013

Link back to DTU Orbit

Citation (APA):

Ye, F., \& Morioka, T. (2013). Interleaved Core Assignment for Bidirectional Transmission in Multi-Core Fibers. In 39th European Conference and Exhibition on Optical Communication (ECOC 2013) (pp. We.2.D.5). IEEE.

\section{General rights}

Copyright and moral rights for the publications made accessible in the public portal are retained by the authors and/or other copyright owners and it is a condition of accessing publications that users recognise and abide by the legal requirements associated with these rights.

- Users may download and print one copy of any publication from the public portal for the purpose of private study or research.

- You may not further distribute the material or use it for any profit-making activity or commercial gain

- You may freely distribute the URL identifying the publication in the public portal

If you believe that this document breaches copyright please contact us providing details, and we will remove access to the work immediately and investigate your claim 


\title{
Interleaved Core Assignment for Bidirectional Transmission in Multi-Core Fibers
}

\author{
Feihong Ye, Toshio Morioka \\ DTU Fotonik, Department of Photonics Engineering, Technical University of Denmark \\ DK-2800 Kgs. Lyngby, Denmark, $\bowtie$ feye@fotonik.dtu.dk
}

\begin{abstract}
We study interleaved core assignment for bidirectional transmission in multi-core fibers. By combining it with heterogeneous core structure in an 18-core fiber, the transmission distance is extended by 10 times compared to homogeneous core structure with unidirectional transmission, achieving a total capacity of $1 \mathrm{~Pb} / \mathrm{s}$ per direction.
\end{abstract}

\section{Introduction}

The capacity of single-mode fibers (SMFs) is ultimately limited by fiber nonlinearities ${ }^{1,2}$ and fiber fuse. Space-division multiplexing (SDM) is a promising candidate to overcome the capacity limit of SMFs so as to sustain the rapid Internet traffic growth ${ }^{3}$. SDM based on uncoupled multi-core fibers (MCFs) is a simple and robust option.

The heterogeneous ${ }^{4}$ and trench-assisted ${ }^{5} \mathrm{MCF}$ structures have been proposed to reduce the intercore crosstalk (XT), thus allowing to increase the core density by adopting a smaller core pitch $\Lambda$. Previously, it was assumed that the signals in different cores of an MCF propagate in the same direction, thus the XT is caused by unidirectional coupling from other cores. To further reduce the XT, bidirectional transmission between neighboring cores in MCFs was proposed ${ }^{6}$. However, the optimization of the core assignment for bidirectional transmission in MCFs has not been addressed yet.

In this paper, we study interleaved core assignment for bidirectional transmission in MCFs. The XT for each core in homogeneous MCFs can be reduced by $50 \%$ compared to unidirectional transmission. We also show that, by applying the interleaved core assignment in heterogeneous MCFs with three non-identical cores, the total XT for each core is reduced by $90 \%$ (or $10 \mathrm{~dB}$ ) compared to unidirectional transmission in homogeneous MCFs. In other words, for the same acceptable XT, the transmission distance in a heterogeneous 18-core fiber could be extended by 10 times compared to a homogeneous MCF with unidirectional transmission, reaching a total capacity of $1 \mathrm{~Pb} / \mathrm{s}$ for each direction.

\section{Bidirectional transmission in MCFs}

Recently, bidirectional transmission of signals in MCFs has been proposed to reduce the $\mathrm{XT}^{6}$. The principle of unidirectional and bidirectional signal propagation and coupling (or the inter-core crosstalk) is illustrated in Fig. 1. By transmitting the signal in neighboring cores of an MCF bidirectionally, the inter-core crosstalk can be reduced significantly compared to unidirectional propagation. One of the other features about bidirectional transmis- sion is that it can be combined easily with other techniques proposed previously for XT reduction, such as heterogeneous ${ }^{4}$ and trench-assisted ${ }^{5} \mathrm{MCF}$ structures. The bidirectional transmission scheme shown here is for the case of 2 cores in an MCF. To apply this technique in MCFs with more cores, an optimization of the core assignment is needed, which has never been reported to date.

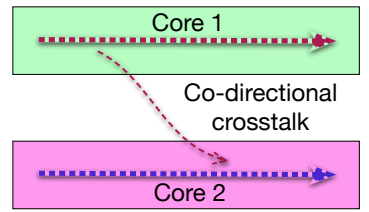

(a)

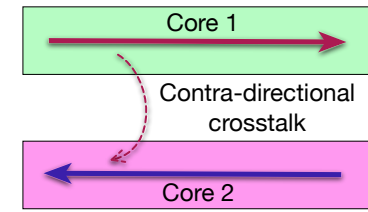

(b)
Fig. 1: Signal propagation and crosstalk coupling in two neighboring cores of an MCF by (a) unidirectional and (b) bidirectional transmission.

\section{Interleaving in homogeneous MCFs}

The most popular structure used to arrange the cores in MCFs is the hexagonal close-packed structure (HCPS), as shown in Fig. 2(a). The main issue with the HCPS is that the center core experiences excessive crosstalk compared to the outer cores ${ }^{7}$. Other structures have been proposed to eliminate this issue, including two-pitch structure (TPS) ${ }^{8}$ and onering structure (ORS) ${ }^{9}$, as shown in Fig. 2(b) and Fig. 2(c), respectively. With bidirectional transmission, the propagation directions in the cores of a homogeneous MCF (all cores have the same refractive index profiles) for different structures can be assigned in an interleaved manner as shown in Fig. 2.

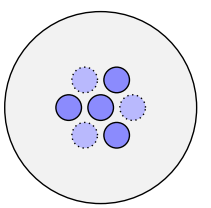

(a)

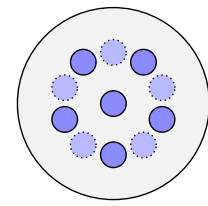

(b)

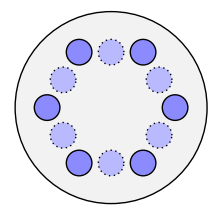

(c)
Fig. 2: Interleaved bidirectional assignment in the cores of MCFs with (a) hexagonal close-packed structure, (b) two-pitch structure and (c) one-ring structure, where the cores with solid (dark color) and dotted (light color) boundaries are for forward and backward signal propagations, respectively. 
For homogeneous MCFs with a small bending radius, the mean crosstalk between two adjacent cores can be analytically expressed as ${ }^{10}$

$$
X T_{\mu}=\frac{2 k^{2} R_{\mathrm{b}}}{\beta \Lambda} \cdot L,
$$

where $k$ is the mode coupling coefficient, $R_{\mathrm{b}}$ is the bending radius, $\beta$ is the propagation constant and $L$ is the total fiber length.

It is assumed that the pitch between adjacent cores is the same for all the three structures as in Fig. 2. For unidirectional transmission with a fixed fiber length, if the pitch between two adjacent cores is $\Lambda_{\mathrm{o}}$, and the XT between these cores is denoted as $X T_{\mathrm{o}}$, the total XT for the center core in HCPS will be $6 X T_{0}$. Since the $X T_{\mu}$ is directly related to pitch, the XT from non-neighboring cores can also be estimated by Eq. (1). The value of total XT for the cores in different structures with interleaved bidirectional assignment is listed in Table 1. Only the crosstalk from the other cores where the signals are propagating in the same direction is included since the contra-directional XT is $17 \mathrm{~dB}$ smaller compared to co-directional XT at a fiber length of $98 \mathrm{~km}^{6}$. For HCPS and TPS, the outer cores with the same propagation direction as the center core suffer more XT compared to the other outer cores with opposite propagation direction. For ORS, the XT of the outer cores have two different values since the spacing between the cores of forward and backward propagation directions is different. The total XT is reduced by half for the center core in both HCPS and TPS, and more than half for the outer cores compared to unidirectional transmission in homogeneous MCFs.

Table 1: Total inter-core crosstalk (normalized to $X T_{\mathrm{o}}$ ) for the cores in MCFs with bidirectional assignment.

\begin{tabular}{c|c|c}
\hline Structures & Center core & Outer cores \\
\hline \hline HCPS & 3 & 1.2 or 2.2 \\
TPS & 3.1 & 1.7 or 2.3 \\
ORS & NA & 1.9 or 2.1 \\
\hline
\end{tabular}

The HCPS, TPS and ORS in Fig. 2 only accommodate 7, 11 and 12 cores in an MCF, respectively. As the number of cores increases, the arrangement of the cores will be spread over more than one ring $^{11}$, as shown in Fig. 3(a), where the two blue dashed circles denote the rings, counting from the innermost one as the first. In order to accommodate as many cores as possible, the HCPS is used when the cores spread over multiple rings, but without the center core due to the excessive XT issue.

To fully utilize the benefits of bidirectional transmission, the number of cores with opposite propagating directions should be the same. Since there is no core in the center and an even number of cores in the rings, the total number of cores in such an MCF will be even. With an aim of equal bidirectional assignment, the circular core-interleaved structure as shown in Fig. 3(c) is proposed. In each ring, the signals in neighboring cores are propagating contradirectionally. The radial ring-interleaved structure shown in Fig. 3(b) is presented here for comparison. In this structure, the signals in all the cores of the same ring are propagating in the same direction, but the signals in the cores of neighboring rings are propagating in opposite directions. The total XT for the cores of the MCF structures represented in Fig. 3 is listed in Table 2.

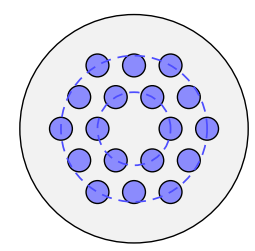

(a)

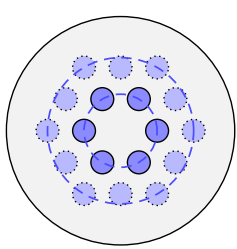

(b)

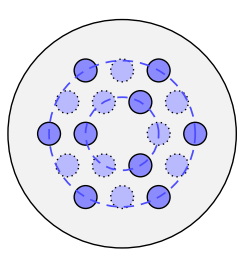

(c)
Fig. 3: Multiple rings of cores in MCFs with (a) unidirectional, (b) radial ring-interleaved and (c) circular core-interleaved bidirectional assignments.

Table 2: Total inter-core crosstalk (normalized to $X T_{\mathrm{o}}$ ) for the cores of 18-core MCFs with unidirectional and bidirectional assignments.

\begin{tabular}{c|c|c|c}
\hline Cases & 1st ring & 2nd ring & choices \\
\hline \hline Fig. 3(a) & 10.7 & 8.4 or 9.2 & $\times$ \\
Fig. 3(b) & 3.7 & 5.2 or 5.4 & $\times$ \\
Fig. 3(c) & 4.4 or 4.9 & $3.3,3.6$ or 4.0 & $\checkmark$ \\
\hline
\end{tabular}

With the circular core-interleaved bidirectional assignment, the total crosstalk for each core in homogeneous MCFs can be reduced by more than half. The overall crosstalk reduction performance in this structure is better than in the radial ring-interleaved structure, and is thus preferred. Since the HCPS is used, not all the cores in the $2^{\text {nd }}$ ring are at the same distance to the center of the MCF.

\section{Interleaving in heterogeneous MCFs}

To further reduce the XT, the heterogeneous structures, where the cores have different refractive index profiles, can be used together with interleaved bidirectional assignment. As shown in Fig. 4, three nonidentical cores (differentiated by colors) are used as heterogeneous cores in an MCF, where all the cores in the $2^{\text {nd }}$ ring are at the same distance to the center of the MCF. Fig. 4(b) shows the interleaved core assignment, where the signals propagating direction in the blue cores in the $2^{\text {nd }}$ ring are determined by the circular core-interleaved structure while for the red and yellow cores in the $1^{\text {st }}$ and $2^{\text {nd }}$ rings, the ringinterleaved structure is applied. The corresponding total crosstalk of each core in a heterogeneous MCF with unidirectional and interleaved bidirectional assignments is listed in Table 3. 


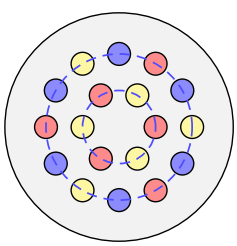

(a)

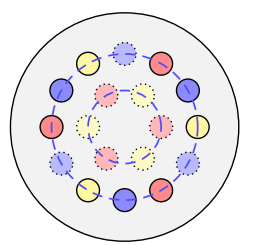

(b)

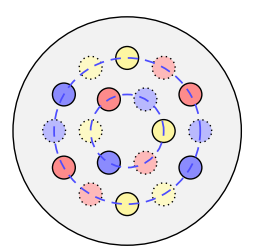

(c)
Fig. 4: (a) Unidirectional, (b) interleaved and (c) improved interleaved bidirectional assignments in heterogeneous MCFs with three non-identical cores.

Table 3: Total inter-core crosstalk (normalized to $X T_{\mathrm{o}}$ ) for the cores in heterogeneous MCFs using unidirectional and bidirectional assignments.

\begin{tabular}{c|c|c|c}
\hline Cases & 1st ring & 2nd ring & choices \\
\hline \hline Fig. 4(a) & 2.6 & 2.1 & $\times$ \\
Fig. 4(b) & 1.2 & 0.6 & $\times$ \\
Fig. 4(c) & 0.95 & 0.6 & $\checkmark$ \\
\hline
\end{tabular}

The issue with interleaved bidirectional assignment in heterogeneous MCFs, as shown in Fig. 4(b), is that the $\mathrm{XT}$ for the cores in the $1^{\text {st }}$ ring is almost twice that of the ones in the $2^{\text {nd }}$ ring. An improved version is proposed in Fig. 4(c), where the circular core-interleaved bidirectional assignment is used. The three non-identical cores are also arranged circularly, thus, the number of non-identical cores is the same in each ring.

\section{Discussions}

The normalized total crosstalk in each core of MCFs with three assignments is plotted in Fig. 5, where the square, circle and triangle shapes are for unidirectional (as in Fig. 3(a)), interleaved bidirectional (as in Fig. 3(c)) assignments in homogeneous MCFs and interleaved bidirectional assignment in heterogeneous MCFs (as in Fig. 4(c)), respectively.

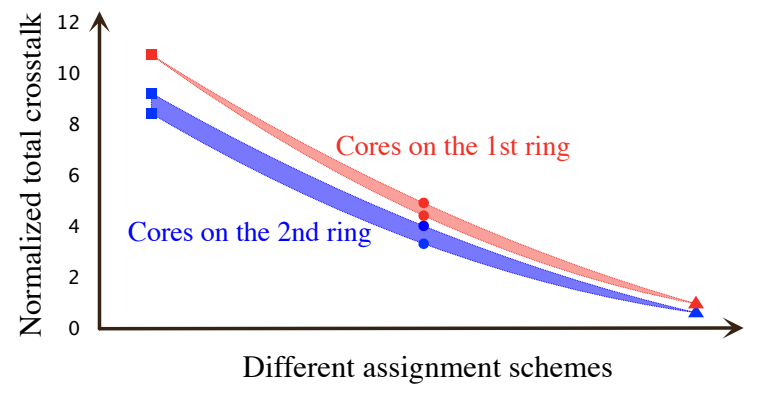

Fig. 5: Normalized total crosstalk of each core in different rings with three assignment schemes.

By using bidirectional assignment in HCPS arranged homogeneous 18-core MCFs, the XT of each core is reduced by more than half. By applying interleaved bidirectional assignment in heterogeneous 18-core MCFs with three non-identical cores, the $\mathrm{XT}$ of each core in the $1^{\text {st }}$ and the $2^{\text {nd }}$ rings is only $1 / 11$ and $1 / 14 \sim 1 / 15$ of that for the case of unidirectional homogeneous 18-core MCFs, respectively.
As shown in Ref. ${ }^{12}$, a trench-assisted HCPS arranged homogeneous 19 -core MCF of $10.1 \mathrm{~km}$ was fabricated, with cladding diameter, cladding thickness and core pitch of $200 \mu \mathrm{m}, 30 \mu \mathrm{m}$ and $35 \mu \mathrm{m}$, respectively. The total average XT per length for each core is $-42 \mathrm{~dB} / \mathrm{km}$, with a total average XT of $-32 \mathrm{~dB}$ for each core over the whole fiber link. With interleaved bidirectional assignment and heterogeneous core structure, the transmission distance can be extended by 10 times to $101 \mathrm{~km}$ while keeping the total XT at $-32 \mathrm{~dB}$ for each core unchanged, since the total XT for each core is less than $10 \%$ of that for the unidirectional homogeneous scheme. Due to the fiber nonlinear limit, the maximum capacity of each core in single-mode operation within one span $(100 \mathrm{~km})$ is around $110 \mathrm{~Tb} / \mathrm{s}$ when using polarization-division multiplexing (PDM) and the 32QAM modulation format, where the XT should be lower than $-30 \mathrm{~dB}$ for practical application ${ }^{11}$. With a total XT for each core of $-32 \mathrm{~dB}$ over a $101 \mathrm{~km}$ fiber length, a total capacity of $1 \mathrm{~Pb} / \mathrm{s}$ per direction can be anticipated for a heterogeneous 18core fiber with interleaved bidirectional assignment.

\section{Conclusions}

We have studied the interleaved core assignment for bidirectional transmission to reduce inter-core crosstalk in MCFs. With interleaved bidirectional assignment, the crosstalk suffered by each core can be reduced by more than half. In other words, the core density can be doubled if the crosstalk is kept identical to the case with unidirectional transmission. By applying the interleaved bidirectional assignment in heterogeneous 18 -core fibers with three non-identical cores, the total XT of each core is only $7 \% \sim 9 \%$ of that for unidirectional homogeneous MCFs. Thus, the improved bidirectional assignment scheme has the potential to effectively extend the transmission distance of MCFs. For example, the transmission distance in a trenched-assisted homogeneous 18-core fiber could be extended from 10.1 to $101 \mathrm{~km}$ by using a heterogeneous structure and interleaved bidirectional assignment together, while keeping the XT at $-32 \mathrm{~dB}$ for each core, achieving a total capacity of $1 \mathrm{~Pb} / \mathrm{s}$ for each direction.

\section{References}

[1] R.-J. Essiambre et al., Proc. IEEE, 100, 1035-1055, 2012.

[2] G. Bosco et al., Opt. Express, 19, B438-B449, 2011.

[3] T. Morioka, OECC 2009, FT4.

[4] M. Koshiba et al., IEICE Electron. Express., 6, 98-103, 2009.

[5] K. Takenaga et al., OFC 2011, OWJ4.

[6] T. Ito et al., OFC 2013, OTh3K.2.

[7] S. Matsuo et al., OFC 2013, OM3I.3.

[8] S. Matsuo et al., Opt. Lett., 36, 4626-4628, 2011.

[9] S. Matsuo et al., Opt. Express, 20, 28398-28408, 2012.

[10] M. Koshiba et al., IEEE Photonics Journal, 4, 1987-1995, 2012.

[11] F. Ye et al., to be presented at OECC/PS 2013, WR2-3.

[12] J. Sakaguchi et al., J. Lightwave Technol., 31, 554-562, 2013. 